\title{
Nonequilibrium aspects in relativistic nuclear collisions
}

\author{
J. Cugnon \\ W. K. Kellogg Radiation Laboratory, California Institute of Technology, Pasadena, California 91125 \\ and Physics Department, University of Liege, B-4000 Sart-Tilman, Belgium \\ (Received 29 August 1980)
}

\begin{abstract}
The possibility of splitting the inclusive cross section for relativistic nuclear collisions into a direct and a thermal component is examined. It is pointed out that the definition of a direct term is model dependent. Besides this difficulty, it is explained why two particle correlation measurements can give ambiguous information on the size of the direct component. The decomposition of the inclusive cross section in the context of Monte Carlo calculations, according to the number $n$ of collisions undergone by the ejected nucleons, is considered. Numerical results for $\mathrm{Ne}+\mathrm{Ne}$ and $\mathrm{Ca}+\mathrm{Ca}$ at $E / A=800 \mathrm{MeV}$ show that the cross sections cannot be split into two parts that could be regarded as a direct and a thermal part, but rather indicate a continuously rising departure from equilibrium as $n$ decreases. Dominance of some values of $n$ in some regions of the momentum space is exhibited. The $\Delta$-particle spectra show a completely different pattern and look similar for all values of $n$.
\end{abstract}

[NUCLEAR REACTIONS Relativistic nucleus-nucleus collisions. Direct and thermal contributions. Nonequilibrium intermediate processes. ${ }^{20} \mathrm{Ne}+{ }^{20} \mathrm{Ne}$, ${ }^{40} \mathrm{Ca}+{ }^{40} \mathrm{Ca} . E / A=800 \mathrm{MeV}$.

\section{INTRODUCTION}

There has recently been an important discussion on the question of the relative dominance of the direct and the thermal processes to the nucleon emission in relativistic nuclear collisions. ${ }^{1-3}$ We would like to clarify the issue in relation to recent Monte Carlo calculations, ${ }^{4,5}$ which include from the very beginning direct and multiple scattering processes on an equal footing.

In Sec. II, we briefly review the status of the question and emphasize that the two components are not uniquely defined. Once the definition of the direct component has been chosen, the question arises as to whether the other component is of a thermal type or not. We analyze how some specific models, essentially three- and one-dimensional cascade models, can help in answering this question and show that two possible decompositions of the inclusive cross sections are very useful in this respect. We point out a difficulty in extracting reliable information about the direct component, when properly chosen, from two nucleon correlation measurements. In Sec. III, we consider numerically the decomposition of the inclusive cross section, in the context of our Monte Carlo calculations, according to the number of collisions suffered by the nucleons. We show that the inclusive cross section cannot be decomposed into a direct and a thermal part. We also consider the same question for the $\Delta$ particles (or the emitted pions in our model). The latter shows a completely different behavior. Finally, Sec. IV contains our conclusion.

\section{DECOMPOSITION OF THE INCLUSIVE CROSS SECTIONS}

\author{
A. Decomposition into "direct" and "thermal" \\ components
}

The gross features of the proton inclusive cross section in the GeV range have been reproduced in an equally acceptable manner by the fireball ${ }^{6,7}$ and the so-called direct (or clear) knockout ${ }^{8}$ models. Then, rather naturally, came the idea that the inclusive cross section is the sum of a direct and a thermal component

$$
\sigma=\sigma_{\mathrm{D}}+\sigma_{\mathrm{Th}} \text {. }
$$

Recently ${ }^{1,2,9}$ people have tried to determine the relative importance of the two components, basing their investigation on the validity of Eq. (2.1). We would like to stress that these two quantities are not uniquely defined. A model is needed to define at least one of them [the other one can be obtained by subtraction if Eq. (2.1) holds]. Usually, the direct component is calculated with a formula corresponding to the graph of Fig. 1(a), which involves the nuclear vertex function and the two nucleon collision cross section. This definition of the direct component seems very reasonable, but its evaluation is blurred by two main uncertainties: (a) The nuclear vertex function, usually taken as the nucleon momentum distribution, is not known in detail. (b) The nucleonnucleon cross section should be corrected for off-shell effects, of which our knowledge is not complete.

The clear knockout process [Fig. 1(a)] is by no 


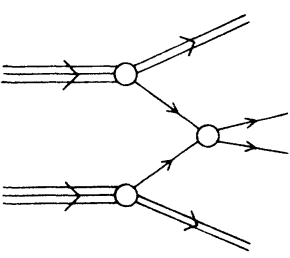

(a)

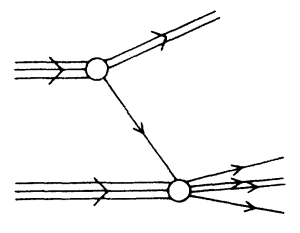

(b)
FIG. 1. Possible diagrams associated with a direct component (see text).

means the unique choice for $\sigma_{\mathrm{D}}$. An alternative choice is provided by the graph of Fig. 1(b), which might be of interest, as we discuss below.

Our main purpose here is to study the validity of relation (2.1). Whatever definition of the direct process is chosen, this validity demands that the remaining part of the inclusive cross section has a thermal nature, i.e., is isotropic and equilibrated. There is no a priori reason for this to be so and a phenomenon similar to the precompound emission might occur: For nucleon and deuteron induced reactions at energy larger than $\sim 10 \mathrm{MeV}$, a third component appears in the spectrum of the emitted particles and fills the gap between the direct (high energy) part and the compound nucleus or evaporation (low energy) part (for a review, see Refs. 10, 11).

\section{B. Decomposition into clusters or in terms of collision number}

A convenient method for studying the plausibility of a decomposition such as (2.1) is provided by the Monte Carlo cascade models. Within this context, the inclusive cross section can be decomposed in a series of terms and a study of the properties of these different terms could determine whether they can be regrouped into two terms that can be regarded as direct and thermal components. A possible decomposition is performed according to the number of collisions suffered by the outgoing nucleons:

$$
\sigma=\sigma_{1}+\sigma_{2}+\sigma_{3}+\sigma_{4}+\cdots,
$$

where $\sigma_{n}$ is the (differential) cross section for ejecting a nucleon after it has undergone $n$ collisions. ${ }^{12}$ Another possible decomposition has been put forward by the "rows on rows" model ${ }^{13,14}$ and is obtained by noticing that two-baryon collisions link $M$ particles of the projectile with $N$ particles of the target:

$$
\sigma=\sum_{M_{0} N} \sigma_{M N},
$$

where the dependence upon $\overrightarrow{\mathrm{p}}$ has been made implicit (that is not important for our discussion here).
In expansion (2.3), the direct process can be represented by $\sigma_{11}$ which in turn can be identified with the clear knockout process of Fig. 1(a) (except for quantal off-shell correction). The question then arises as to whether the rest of the series (2.3) can be considered as a thermal component. Two conditions are to be met: (i) the resulting spectrum should be thermal-like; (ii) spectra for small values of $M, N$ should not be too similar to $\sigma_{11}$, or in other words, there must be a sharp transition between $\sigma_{11}$ and the spectrum for neighboring values of the pair $(M, N)$. However, there is no reason to identify the direct component with $\sigma_{11}$ solely and one could look for a better splitting of the series (2.3), which would satisfy the previous criteria. This, however, does not seem to be the case with the linear cascade model. ${ }^{13}$ The question is presently investigated in the context of a three-dimensional cascade calculation. ${ }^{15}$

One can as well address the question of whether Eq. (2.2) can be split into two pieces that can be interpreted as direct and thermal components. The first term $\sigma_{1}$ cannot then be associated with the diagram of Fig. 1(a). This, however, has been done in the literature ${ }^{1,16}$ and adds to the confusion. Rather, $\sigma_{1}$ should be associated with diagram of Fig. 1(b) (provided the second bubble is calculated in the impulse approximation). This diagram provides another acceptable definition of the direct process. Not very much attention has been paid to the question of how much this diagram can contribute to the inclusive cross section. However, a similar diagram has been studied in relation to the scaling in the forward direction. ${ }^{17,18}$

Once again, the same question can be addressed concerning $\sigma_{1}$ : Can the rest of the sum (2.2) be considered as a thermal component? In Sec. III, we investigate this problem numerically in the context of our recent Monte Carlo calculations. ${ }^{4,5}$

Before closing this section, we would like to draw attention to an aspect of the problem which has been overlooked and indicates that two-nucleon correlation measurements cannot really give us relevant information about $\sigma_{D}$, even if we have decided what is the definition of $\sigma_{\mathrm{D}}$. Let us consider expansion (2.2) for instance, which we rewrite as

$$
\sigma=\sigma_{1}+\sigma_{r},
$$

and let us assume, for a while, that $\sigma_{1}$ and $\sigma_{r}$ can be identified as $\sigma_{\mathrm{D}}$ and $\sigma_{\mathrm{Th}}$. Then the two particle inclusive cross section (or correlation yield) is given by (we indicate here the momentum dependence, for clarity) 


$$
\begin{aligned}
\sigma^{(2)}\left(\overrightarrow{\mathrm{p}}_{1}, \overrightarrow{\mathrm{p}}_{2}\right) & =\sigma_{1} P_{1}\left(\overrightarrow{\mathrm{p}}_{1}, \overrightarrow{\mathrm{p}}_{2}\right)+\sigma_{r} P_{r}\left(\overrightarrow{\mathrm{p}}_{1}, \overrightarrow{\mathrm{p}}_{2}\right) \\
& +\tilde{\sigma} P\left(\overrightarrow{\mathrm{p}}_{1}, \overrightarrow{\mathrm{p}}_{2}\right) .
\end{aligned}
$$

This equation simply embodies the observation that the two detected particles could have been emitted both through a direct process, or both through a multiple collision process, or one through a direct collision and the other through multiple scattering. The quantity $P_{1}\left(\overrightarrow{\mathrm{p}}_{1}, \overrightarrow{\mathrm{p}}_{2}\right)$ [or $P_{r}\left(\overrightarrow{\mathrm{p}}_{1}, \overrightarrow{\mathrm{p}}_{2}\right)$, or $\left.\tilde{P}\left(\overrightarrow{\mathrm{p}}_{1}, \overrightarrow{\mathrm{p}}_{2}\right)\right]$ is the probability of having two particles emitted with the appropriate kinematics from a direct (or multiple scattering, or both) process. A decomposition such as (2.4) also holds if we choose $\sigma_{11}$ as the direct component. In these cases, $\tilde{\sigma}$ can be expressed in terms of the $\sigma_{M N}$ 's and quantities $\sigma\left(M N ; M^{\prime} N^{\prime}\right)$ which are proportional to the probability of having in the same event a cluster of type $(M, N)$ and a cluster of type $\left(M^{\prime} N^{\prime}\right) .^{14}$ Let us stress that the third term does not come from quantum interference [all of the equations (2.1)-(2.4) neglect quantum interference], but rather from the possible coexistence of two different mechanisms involving different parts of the system in the same event.

As a consequence of Eq. (2.4), correlation measurements cannot $a$ priori give valuable information upon the decomposition (2.3), unless one makes a specific assumption upon $\tilde{\sigma}$. In fact, that is what is done in Refs. 1 and 9. In particular, it is assumed that the probability of one proton coming from a direct process while the other is coming from a multiple scattering process vanishes. Note that assuming $\tilde{P}=P_{r}$ would determine the ratio $\sigma_{1} /\left(\sigma_{r}+\tilde{\sigma}\right)$ instead of $\sigma_{1} / \sigma_{r}$.

Let us finally mention that expansions (2.2) and (2.3) cannot be obtained from one another. In particular, $\sigma_{11}$ is not equal to $\sigma_{1}$. Instead, $\sigma_{11} \leqslant \sigma_{1}$.

\section{PROPERTIES OF THE CROSS SECTIONS $\sigma_{i}$}

We have calculated these cross sections with our Monte Carlo code, which is described extensively in Refs. 4 and 5 . It is sufficient to say here that we describe the collision process as a succession of relativistic, classical, on-shell baryon-baryon collisions. Pion production is taken into account by introducing the possibility of creating $\Delta$ particles. The latter can disappear through the $N \Delta \rightarrow N N$ reaction and scatter on nucleons and other $\Delta$ 's. They are assumed to decay and release pions at the end of the strong interaction process, i.e., when the chain of binary collisions ceases. This model is very successful in reproducing one- and two-nucleon inclusive data. ${ }^{5}$

We first discuss the numerical values of the integrated cross sections (over the invariant phase space). Those quantities are related to the total reaction cross section $\sigma_{R}^{\text {tot }}$ by

$$
\sum_{n} \sigma_{n}=\sigma_{R}^{\text {tot }} \bar{\nu}
$$

where $\bar{\nu}$ is the average (over the impact parameter) number of participant nucleons. It is also useful to define the frequency $f(n)$, i.e., the number of nucleons which are ejected after $n$ collisions. For any impact parameter $b$, we have

$$
\sum_{n} f(n)=\nu(b)
$$

The average (over impact parameter) value of $f(n)$ are related to the $\sigma_{n}$ 's by

$$
\overline{f(n)}=\bar{\nu} \frac{\sigma_{n}}{\sum_{n} \sigma_{n}}=\frac{\sigma_{n}}{\sigma_{R}^{\text {tot }}} .
$$

The numerical values of these quantities are presented for $\mathrm{Ne}+\mathrm{Ne}$ and $\mathrm{Ca}+\mathrm{Ca}$ at $E / A=0.8 \mathrm{GeV}$ on the rhs of Fig. 2. The rest of the figure displays the frequency $f(n)$ for central and peripheral collisions. Several observations are relevant. First, the distribution of the frequencies $\overline{f(n)}$ roughly scales as $A$, the total mass number of the system. A slight deviation from this scaling occurs for central collisions. The distribution has a longer tail for $\mathrm{Ca}+\mathrm{Ca}$, which, of course, comes from the larger linear dimension of this system. Second, for central collisions, the nucleons have an equal chance to make 1,2 , or 3 collisions, whereas for peripheral collisions, most of the participant nucleons make only one

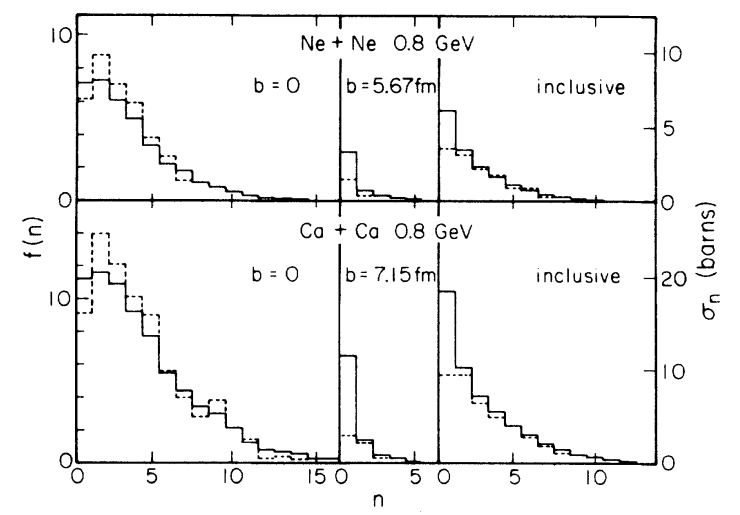

FIG. 2. Probability of the ejection of nucleons (solid lines) after $n$ collisions. They are normalized as $\Sigma_{n} f(n)$ $=\nu$, the number of participants. The right-hand part of the figure gives the average (over impact parameter) freauency $\overline{f(n)}$ (scale on the left) as well as the cross section $\sigma_{n}$ (scale on the right). See text for more details. The dotted lines give the same probability for the $\Delta^{\prime}$ s. They are normalized as $\Sigma_{n} f(n)=8 \nu_{\Delta}, \nu_{\Delta}$ being the number of $\Delta$ 's. 
collision. This obviously reflects the geometrical properites of the participant system. Finally, the frequency distribution for the $\Delta$ 's (and of the subsequent pions) closely follows in shape the nucleon frequency distribution, except for $n=1$ and $n=2$ and central collisions. The reason is simple. After the first collisions, the produced $\Delta$ 's have a low velocity. Hence, they have little chance to escape the system without being hit by the other nucleons traveling with the (high) incident velocity.

It is notable that the contribution of single scattering processes is rather high: $44 \%$ and $33 \%$ for $\mathrm{Ne}+\mathrm{Ne}$ and $\mathrm{Ca}+\mathrm{Ca}$, respectively. The average values of $n$ are 2.78 and 3.24, respectively.

Figure 3 shows the central result of this paper,

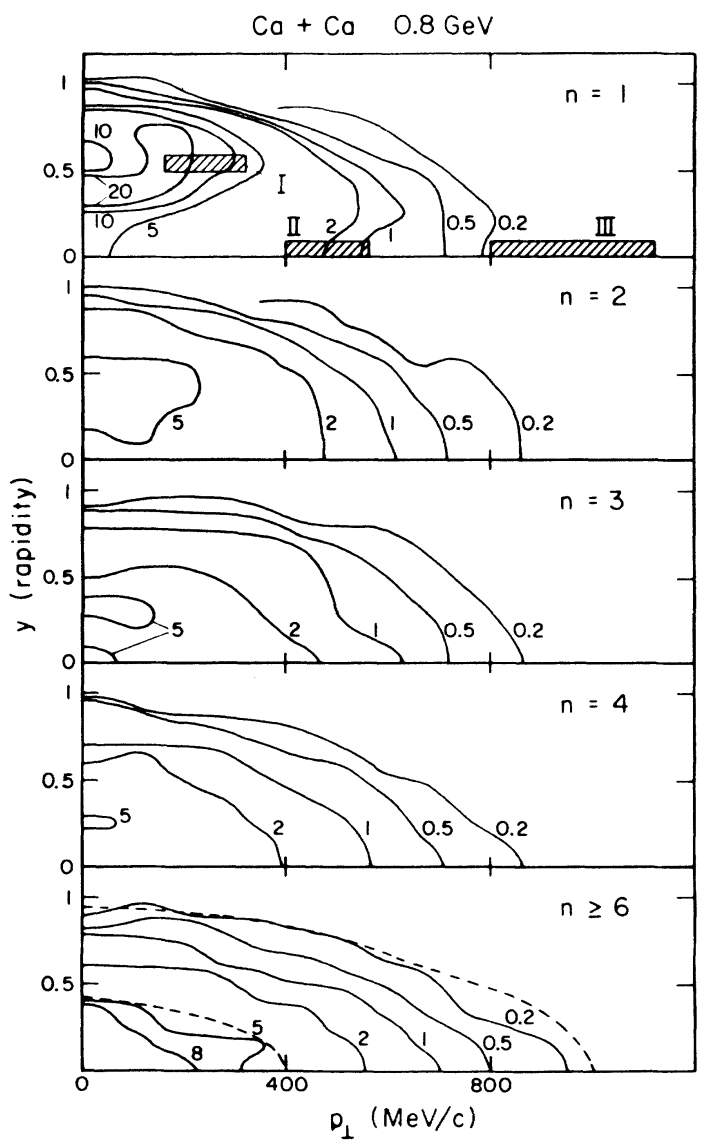

FIG. 3. Invariant cross sections to produce a proton after $n$ collisions as a function of the c.m. perpendicular momentum $p_{1}$ and c.m. rapidity $y$. They are given in units of $0.462 \times 10^{4} \mathrm{mb} \mathrm{c}^{3} \mathrm{GeV}^{-2}$. The regions I, II, III are connected with the content of Table I. To give an idea of the isotropy $n \geqslant 6$, curves of equal energy of 1020 and $1371 \mathrm{MeV}$ (dotted curves) have been drawn. The full curves are smooth interpolations of the results of the Monte Carlo calculation. Those results take the form of numbers defined on mesh points every $\Delta y=0.1$ and $\Delta p_{\perp}=80 \mathrm{MeV} / c$. namely the invariant cross section for ejecting a proton after $n$ collisions. We show only the result for $\mathrm{Ca}+\mathrm{Ca}$, the one for $\mathrm{Ne}+\mathrm{Ne}$ being qualitatively the same. Clearly there is a continuous transition between $n=1$ and $n \geqslant 6$, for which the spectrum is fairly, but not completely (see Fig. 4 ), isotropic. The $n=1$ cross section is largely dominated by the $N-N$ cross section, which, folded with the Fermi motion, would create a peak at $p_{\perp}=0$ and $y=0.61$, the incident rapidity in the c.m. system. A ridge would start from that point and extend along the line of equal energy passing through it. So a saddle point would be expected in the vicinity of $y=0, p_{\perp}=600 \mathrm{MeV} / c$. The deviations from this pattern are due to: (a) the fact that the projectile (target) nucleons which are ejected after their first collisions do not exclusively collide with the unperturbed target (projectile) Fermi sphere, but do collide with nucleons which may have made collisions previously; (b) the delta production, which, because of the inelasticity, tends to populate the midrapidity region $\left(y \approx 0, p_{\perp} \approx 0\right)$. We checked this point by repeating the calculation and switching off the $\Delta$ production. In that case, a minimum appears at the origin in the $y-p_{\perp}$ plane.

It has been conjectured ${ }^{1}$ and often stated that the high $p_{\perp}$ region at $90^{\circ} \mathrm{c} . \mathrm{m}$. can be only populated by multiple scattering, whereas the region at small $p_{\perp}$ and at rapidity close to the projectile or target rapidity is overwhelmingly populated by single scattering. Consequently, the region at moderate $p_{\perp}$ and zero rapidity in the c.m. is expected to be populated by both processes. Our results show that this conjecture is qualitatively correct. To quantify this comparison, we have shown in Table I the relative importance of the $n$-collision processes for the three shaded areas which appear in Fig. 3. The only surprising result, at least in our opinion, is that region III does not correspond solely to high values of $n$. Already a two collision process populates almost as much as the $n \geqslant 6$ component.

The smooth change of the spectra with the parameter $n$ is dramatically shown in Fig. 4, where we have plotted their simplest characteristics, namely their variance along the $p_{\|}$and $p_{\perp}$ variables. There is no discontinuity in the trend which would allow a separation into a direct and a thermal contribution.

It is instructive, at this point, to compare our results with those of Randrup, ${ }^{19}$ who used essentially the same model to study the equilibration between two infinite pieces of nuclear matter initially at a global relative velocity. The main differences thus come from the finite size effects in our case, although there can also be some effect 


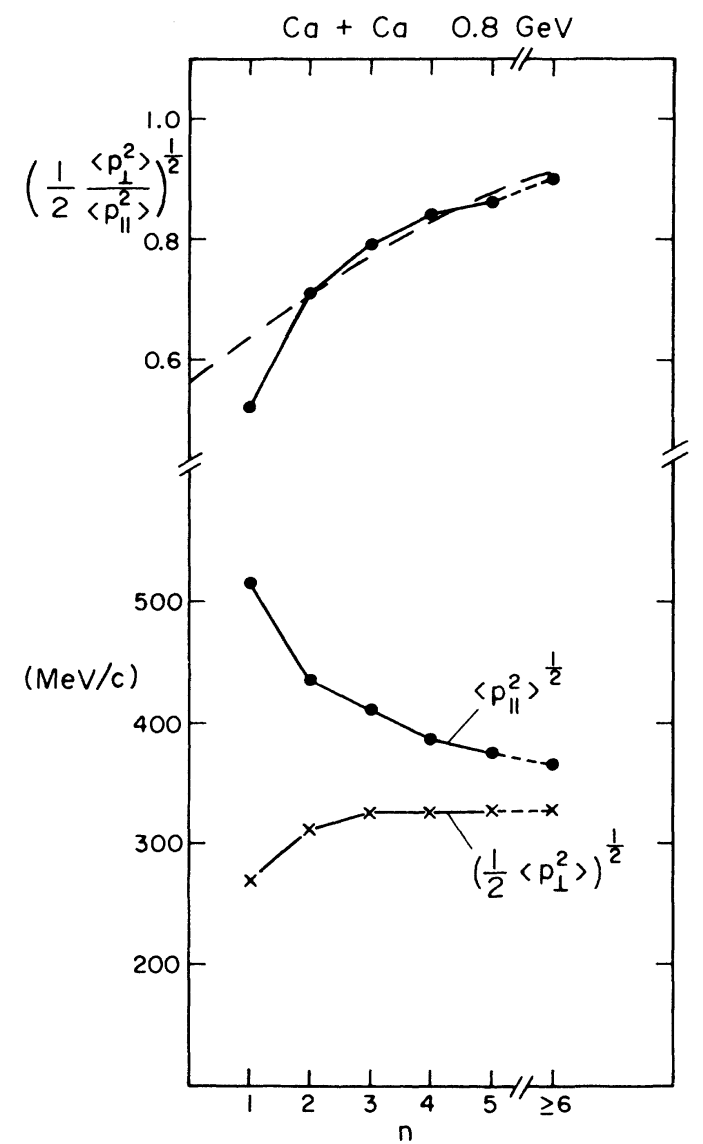

FIG. 4. Parallel and transverse momentum variances, as well as their ratios, of the total c.m. cross sections for producing a nucleon after $\boldsymbol{n}$ collisions. The dashed line indicates the results of Ref. 17 for infinite nuclear matter. This curve, originally given as a function of time, has been rescaled by converting $1 \mathrm{fm} / c$ into 5 units of $n$.

of the compression and subsequent decompression of the matter, which may increase and decrease the frequency of the binary collisions. In Ref. 19, the calculated anisotropy of the system varies with time in a very similar manner as our calculated value (top of Fig. 4) does as a function of $n$. This suggests that the particles suffering $n$ collisions escape from the system at a more or less well defined time. We, unfortunately, cannot check this statement at the present time, but this is certainly a question worth investigating. If we assume this is true, we can consider that the $n \geqslant 6$ component comes from the latest stage of the collision process, say $6-9 \mathrm{fm} / c$ after its beginning. ${ }^{4}$ So, the equilibration time [which can be defined as the time required for the anisotropy of the system (see top of Fig. 4) to pass from its initial value, i.e., $\sim 0$, to a value of 0.90 ] in a system such as $\mathrm{Ca}+\mathrm{Ca}$ is something of this order,
TABLE I. Relative importance (in \%) of $n$-collision processes for the shaded areas of Fig. 3.

\begin{tabular}{lrrrrrr}
\hline \hline & $n=1$ & $n=2$ & $n=3$ & $n=4$ & $n=5$ & $n \geqslant 6$ \\
\hline Region I & 62 & 17 & 7 & 4 & 2 & 8 \\
Region II & 15 & 17 & 15 & 16 & 13 & 24 \\
Region III & 7 & 20 & 17 & 18 & 10 & 28 \\
\hline \hline
\end{tabular}

while Randrup found a considerably shorter time in nuclear matter, $\sim 2 \mathrm{fm} / c$. The finite size effect therefore does seem to play an important role in the equilibration of the system.

Let us finally say a few words about the $\Delta$ particles. Table II shows the frequency at which they are produced as a function of the number of collisions $n$. Except for $n=1$, the ratio of the produced $\Delta$ 's (or pions) to the number of baryons is practically equal to 0.12 . This appears to be the global value for $b=0$ (Ref. 4) and also the asymptotic value for large cluster $\mathrm{s}^{12}$ and thus can be interpreted as an equilibrium value. Table II thus shows that very few collisions are needed to attain chemical equilibrium between $\Delta$ 's and nucleons. We also found that the $\Delta$ 's have fairly isotropic and equilibrated spectra with $\frac{1}{2}\left\langle p_{\perp}^{2}\right\rangle \approx\left\langle p_{\|}^{2}\right\rangle \approx(350$ $\mathrm{MeV} / c)^{2}$ independent of $n$. It is remarkable that this value is approximately equal to the asymptotic value for the nucleons (average of $\left\langle p_{\perp}^{2}\right\rangle / 2$ and $\left\langle p_{11}^{2}\right\rangle$ for large $n$ ). Nonrelativistically speaking, it means that the $n \geqslant 6$ nucleons have a higher "temperature" than the $\Delta$ 's. This situation also occurs in the linear cascade model. ${ }^{10}$ In our opinion, it reflects the fact that the nucleon and the $\Delta$ 's gases interact mainly via the $N N=N+\Delta$ reaction and only weakly through the $N \Delta$ elastic scattering (see also Ref. 4).

\section{CONCLUSION}

We have considered the possibility of decomposing the inclusive cross section in relativistic nucleus-nucleus collisions into a direct and a thermal component. There is no model-independent definition of the direct term and, unfortunately, different authors use different models. We have investigated the possible decomposition of the inclusive cross sections in the context of Monte Carlo calculations and tried to formulate criteria which

TABLE II. Ratio of number of $\Delta$ 's to the number of nucleons as a function of the number of collisions $n$.

\begin{tabular}{ccccccc}
\hline \hline$n$ & 1 & 2 & 3 & 4 & 5 & 6 \\
\hline$N_{\Delta} / N_{B}$ & 0.060 & 0.117 & 0.115 & 0.116 & 0.125 & 0.116 \\
\hline \hline
\end{tabular}


have to be met for those decompositions to reduce to a sum of direct and thermal components. When the cross section is decomposed according to the number of collisions suffered by the ejected nucleons, numerical results show that the criteria are not fulfilled in the $\mathrm{GeV}$ range and that the system exhibits a continuum of nonequilibrium properties. On the other hand, the $\Delta$ particles, which in this model are the source of pion production, are well thermalized, but the $\Delta$ and nucleon components do not appear in mechanical equilibrium.

One may wonder whether the nonequilibrium properties, which are reminiscent of the precompound emission is, like this latter, energy dependent. Indications exist ${ }^{2}$ that in the $250 \mathrm{MeV}$ range, nucleus-nucleus collisions produce protons, whose spectrum might be described by a two-component model [Eq. (2.1)], although, as we discussed in Sec. II, uncertainties are attached to the definitions of the direct and the thermal components.
Let us finally notice that like $\sigma_{\mathrm{D}}$, the $\sigma$ 's are not directly measurable quantities. Nevertheless, analysis along the lines described here is important in the sense that it provides some hint of how the momentum space is populated. This may have an important influence on estimates of the production of composite particles. For instance, nonequilibrium deuteron production is expected in the regions of the momentum space associated with a low value of $n$. An investigation of the deuteron production with this perspective is certainly desirable, as the ratio of deuteron to proton yield is considered as a suitable quantity for testing our views of the reaction process. ${ }^{1,20,21}$

\section{ACKNOWLEDGMENTS}

It is a pleasure to thank Dr. S. E. Koonin for reading the manuscript and for fruitful discussions. We are also grateful to the members of the Kellogg Radiation Laboratory for the hospitality extended to us. This work was supported in part by the National Science Foundation.
${ }^{1} \mathrm{~S}$. Nagamiya, LBL report, 1979.

${ }^{2}$ M. Chemtob and B. Schürmann, Nucl. Phys. A336, 508 (1980).

${ }^{3} \mathrm{~J}$. Knoll, Nucl. Phys. A343, 511 (1980).

${ }^{4} \mathrm{~J}$. Cugnon, T. Mizutani, and J. Vandermeulen, Nucl. Phys. A352, 505 (1981).

${ }^{5} \mathrm{~J}$. Cugnon, Phys. Rev. C 22, 1885 (1980).

${ }^{6} \mathrm{~J}$. Gosset, H. H. Gutbrod, W. G. Meyer, A. M. Poskanzer, A. Sandoval, R. Stock, and G. D. Westfall, Phys. Rev. C 16, 629 (1977).

${ }^{7}$ H. H. Gutbrod LBL Report No. LBL-7766, 1978.

${ }^{8}$ R. L. Hatch and S. E. Koonin, Phys. Lett. $81 \mathrm{~B}, 1$ (1977).

${ }^{9}$ S. Nagamiya et al., J. Phys. Soc. Jpn. Suppl. $\underline{44}, 378$ (1978); Phys. Lett. 81B, 147 (1979); Phys. Rev. Lett. $45,602(1980)$.

${ }^{10} \mathrm{C}$. Mahaux and H. A. Weidenmüller, Annu. Rev. Nucl. Sci. 29,1 (1979).

${ }^{11}$ H. Feshbach, A. Kerman, and S. E. Koonin, Ann. Phys. (N.Y.) 125, 429 (1980).

${ }^{12}$ Decomposition (2.1) is only valid for what we call Monte Carlo calculations, which follow all possible collisions between the nucleons, in contradistinction with some cascade calculations where the nucleons of the projectile are scattered by a target medium. The definition of the number of collisions is rather loose in these calculations.

${ }^{13} \mathrm{~J}$. Hüfner and J. Knoll, Nucl. Phys. A290, 460 (1977).

${ }^{14} \mathrm{~J}$. Knoll and J. Randrup, Nucl. Phys. $\underline{\mathrm{A324}}, 445$ (1979).

15J. Cugnon, J. Knoll, and J. Randrup, Report No. LBL$11301,1980$.

${ }^{16}$ H. J. Pirner and B. Schürmann, Nucl. Phys. A316, 461 (1979).

${ }^{17}$ I. A. Schmidt and R. Blankenbecker, Phys. Rev. D $\underline{15}$, 3321 (1977).

${ }^{18} \mathrm{C}$. Y. Wong and R. Blankenbecker, Phys. Rev. C $\underline{22}$, 2433 (1980).

${ }^{19} \mathrm{~J}$. Randrup, Nucl. Phys. A314, 429 (1979).

${ }^{20}$ P. J. Siemens and J. Kapusta, Phys. Rev. Lett. $\underline{43}$, 1487 (1979).

${ }^{21} \mathrm{H}$. Sato and K. Yazaki, University of Kyoto, report 1980. 